\title{
Evaluating Attitude and Behavior: An Info-Operational Procedure Related/Supported by the Cognitive Centers of Mind
}

\author{
Research Article
}

Volume 2 Issue 1- 2021

\author{
Author Details \\ Florin Gaiseanu* \\ Science and Technology of Information, Bucharest (Romania) and Barcelona (Spain) \\ *Corresponding author \\ Florin Gaiseanu, Science and Technology of Information, Bucharest (Romania) and Barcelona (Spain)
}

Article History

Received: December 21, 2020 Accepted: January 18, 2021 Published: January 20, 2021

\begin{abstract}
In order to highlight the cognitive properties of the mind related with attitude and behavior and their evaluation by a suitable and realistic procedure, in this paper it is analyzed the relation of mind with the body from the informational perspective, showing that the functionality of mind is closely related to the body operability and the mind/body system works on the basis of specialized components and functions, allowing in this way a better operability with higher performances. It is shown that the mind is actually an informational system, working with thought as an informational operator on the accumulated life info-experience field, able to search a specific information in the mind "library" (memory), to order to the motor elements to execute various operations, or to connect the sensorial detectors (senses) to the external or internal world. On this field of information including the judgment criteria, there are received new signals from the internal and external sources, so an adaptive response defined as attitude is returned, expressed as informational output, or retained in memory for further use. Such signals are characteristic of specialized informational sources, determining/managing the affective/emotional info-reactive effects, expressing the body status and health or info-genetic impulses, which are selectively considered by order of priority and/or compared with the existing stable/validated criteria. The evaluation of the final/global attitude is therefore possible by evaluation on a positive/negative (YES/NO) scale of each pro-active partial contributing components resulted from the analysis of the reactive response of each cognitive center. Distinct formulas for the calculation of the momentary attitude and behavior with respect to an object/objective are presented. The obtained results can be applied on a large field of activities, from commercial, financial, educational or health public or private services to political tests of opinion, or on own selfevaluation of personal objectives development. The presented procedure permits also to follow the evolution of attitude on a particular objective during the time.
\end{abstract}

Keywords: Mind/body relation; Informational activities; Cognitive centers; Attitude; Evaluation of attitude and behavior; Infooperational procedure

\begin{abstract}
Abbreviations: CASI: Center of the Acquirement and Storing of Information; IES: Info-Emotional/Affective System; CDC: Center of Decision and Command; GTS: Genetic Transmission System; IC: Info-Connection
\end{abstract}

\section{Introduction}

The attitude is still a debated issue within the psychological field [1], especially because no a description of the mind properties and of the relation with the body was really known till recently [2]. From the traditional point of view and even from the perspective of the modern interested/involved sciences like neurology and neurosciences, the relation of the mind with the body is not yet understood, so the two entities, mind and body, are treated as separated parts of the organism. The recent implication of the science of information in the study of the biologic systems [3], particularly referred to the mind/body problem, brought a new vision on such a relation, highlighting that information is a fundamental component of the body itself, actively participating to the construction/reconstruction of its parts, and which is manifested actually at the mind level by consciousness [4]. As the attitude was regarded to be a "construct" of the mind [1] based on cognition [5], reflecting how the individuals perceive the world [6] and thus characterizing the individual personality [1], it is natural to focus attention on information, on the informational system of 
the human body and on the related cognitive activities. From such a perspective, it is possible to approach in a suitable way how attitude can be evaluated, taking into account the distinct personal character of attitude and its importance not only from the theoretical point of view [5], but also for applicative perspective in educational sciences and even in rehabilitation therapies [7], so the necessity to approach this issue is evident.

With the aim to cover the lack of a really necessary and precise procedure, able to avoid/eliminate/substitute the "vague" concepts on attitude existing and conveyed up to date in this field [8], in this paper there are reported the research results concerning a new evaluation method of attitude and behavior, involving the specific cognitive properties of the mind. Such a general procedure can be applied in industry, commercial and service activities for the evaluation of the customer satisfaction level, in political and social opinion tests and soundings, in education and health evaluation, or even in personal state and projects fulfillment or development.

\section{Analysis of the Mind/Body Relation and Attitude as a Reactive Product of Cognitive Activities}

In a previous article published in the Volume 1, Issue 1 of this journal [9], it is studied and discussed the definition of attitude, which still is an open question, because no full understanding of mind and its properties related with the cognitive activities and with the fundamental functions of the organism were available. Starting from the identification of these functions and their body support, it is possible to detect the real way in which such functions are fulfilled from informational perspective. First of all, it is important to note that the functionality of mind is closely related with the body operability. Secondly, the mind/body system works on the basis of specialized components and functions, allowing in this way a better operability with higher performances, obtained by compartmentalization [3] (organs-type organization and specialized brain connecting zones [10]).

Another observation permitting to detect the specific ranges of activities of the mind, refers to the fact that the mind is actually an informational system, working with thought as an informational operator. Indeed, the activation of a thought is a consequence of a command transmitted to thought to execute a series of info-operations: to search a specific information in the mind "library" (memory), to order to the motor elements to execute a movement, or to connect the sensorial detectors (senses) to the external or internal world. All these operations are actually managed by means of information, so the mind works with pure (virtual) information, recalled from memory and projected on the mental "screen", or perceived from surroundings by means of the body sensors (sight, hearing, tough, taste, smell). On the other hand, the mind receives also internal body signals from the regular functionality, transmitting its needs: hungry, thirst, pain, sexual or other impulses according to the operations of the specialized internal organs. These operability ranges are specific for every person, according to the characteristics inherited form parents and manifested by predispositions and/or typical behavior/attitude in certain typical situations. These signals are interpreted in mind and memorized for shorter or longer periods of time and a reactive command/decision is subsequently transmitted to adequate operational/informational systems supported by the brain for execution.

However, all the operations described above could be also performed by a machine, suitably programmed with a software and equipped with sensors and execution elements. Our computers and laptops, telephone terminals and informational transmission systems of which we enjoy every day are also some usual examples. The microelectronic systems allowed the extraordinary evolution not only of the informational domain itself, but also of the extraordinary evolution of individuals and of the society at the global level. Then, what is the difference between such machines and human operability? Human is provided with sensitive affective sensors, detecting emotions. Human is an affective/emotive creation of nature. Besides the operability according to the activities of own informational system, he is able to feel, to interpret the feelings, to store them in the personal "library" (memory), to use them according to the momentary necessities and to provide a corresponding decision. In one word, the human feels, may and can decide, so the human have an attitude. Besides the capacity to perceive and to decide, affectivity/emotivity is a fundamental component of the human as a social being. And one more fundamental feature: the capability of reproduction.

Any momentary attitude is reported to a perception, either of an object/objective, event or situation, so always the attitude is related with the personal cognitive system, with its operability and with a certain objective, like it can be seen in Fig. 1 central side. Although the same objective is perceived by more than one person, the attitude is individual, every person has his/her attitude, according to the personal characteristics/performances of own informational system and life experience, which offers the specific own reference for the judgment process.

According to the above discussion, the following conclusions can be drawn:

(i) human works with virtual information, coming/perceived from the environment or from own body, which emits signals/ information according to the personal necessities; (ii) the received/ perceived information is captured by the specialized sensors, which are connected to the specialized zones of the brain (memory), provided with corresponding functionality; (iii) the thought is an informational operator which allows to search and operate with the virtual information, related with the active/operative/decisional zones of the brain and with commanded/motor-execution elements; (iv) the automatic operational systems of the body, responsible with the management and execution of the metabolic and reproductive functions collaborate with the mind, transmitting useful signals for further decisional operability, in agreement with the survival necessities; (v) the specialized functions of the organism composing the human mind/body system, participate effectively to the decision.

From these conclusions we have to observe that actually the cognitive functionality of human is operated by the following cognitive centers, defined and named suggestively/synthetically as: Iknow (memory), Iwant (decision), Ilove (affection/emotions), Iam (personal body status), Icreate (creativity/social relations), Icreated (predispositions), Ibelieve (trust), as it is illustrated in the left-central side of Fig.1. Ibelieve represents the cumulus of inherited or acquired educational traits/beliefs from parents and educational factors, which form a stable base of reference criteria. It is evident therefore that Ibelieve intervenes directly in the decisional functionality, providing the reference elements of the judgment/evaluation/decision process. Ibelieve is the stable, fundamental informational base of individuals, allowing the personal selection of new information and its implementation in the informational system. The described functional centers are actually the cognitive centers of the mind, which participates in a direct way to the perception and info-decisional acts, so to the generation of attitude. Finally, the attitude is given and expressed by the center Iwant, which is the informational output of the mind, either by vocal and/corporal language and/or kept in the memory.

According to the above discussion and as it can be observed also from Figure 1 left side, the mind/body relation is achieved by the connections (suggested by arrows) between the supporting informational regions of the brain and corresponding executing organs, so the perception in mind of the body activities by cognitive centers are actually managed by the following informational systems: the center of the acquirement and storing of information (CASI), composed by the sensors and 
specialized brain regions, reflected in mind by the cognitive center Iknow, the center of decision and command (CDC) reflected by Iwant, the info-emotional/affective system (IES) detected by Ilove, the maintenance informational system (metabolic operability) expressed by Iam, the genetic transmission system (GTS) knowable by Icreate, the info-genetic generator, managing the body development/evolution according to the age $[11,12]$ manifested by Icreated, and so-called infoconnection (IC), responsible for info-selection [13,14], interpreted as Ibelieve.

As it can be remarked from the above presentation, the body itself is an intelligent "machine", working with information, manifested not only by the transmission of pertinent signals to the mind according to its necessities, but also operating automatically to drive the mechanical/ chemical/physical processes which assure its existence. Body matter itself ("hardware" of the mind/body system) works in a modular way [15], driven by information, to assure the metabolic processes, the base of the "software" operability of the mind. Therefore, from the informational perspective, the mind/body is a dynamic, selforganized and self-operational/controlled system including the effective operability, auto-motivated by survival, able to adapt itself according to internal and external necessities and cues, the attitude serving to make suitable decisions for adaptation. The affective/ emotional component of the human organism, as well as that of the other living being, is characteristic to associativity and social relations, as a fundamental need of survival, related to the reproduction and life organization, something which does not exist up to date in artificial systems, as much as developed they could be. Ilove and Icreate have therefore social dimensions, characteristic to life

\section{Evaluating Method of Attitude/Behavior and Related Characteristics}

As it was highlighted above, the mind works with information, which is operated with respect to the internal accepted/selectively stored criteria, either acquired especially during the first years from parents and from other educational sources (Icreated), or latter. With respect to a certain criterion, an information can be accepted or rejected, so the operational system of the mind works on the basis of binary YES/NO mechanisms, like the computers. On these bases, the information is selected and processed to return a decision, which is an output information, i.e. attitude. The selection and info-operation is actually a comparative judgment, an evaluation of the perceived reality, specifically of an object/objective, according to own criteria. The selected information is classified in Good/Bad (YES/NO) sorts, stored in memory to be used as criteria in further decisional processes on the informational "logic" chain like in computers, in concordance with the necessities, and/or explicitly expressed. Therefore, a focused/ perceived "thing"/think as an object/objective of attention is analyzed and categorized as Good/Bad and accepted (YES) or rejected (NO) in a binary elemental mode.

On the other hand, it was shown in an article published in the same issue of this journal [9] that the attitude is expressed through the center Iwant, as a function of all other cognitive centers, participating actively to the final output decisional process. This is a natural consequence of the fact that the mind evaluates globally a certain situation, engaging a momentary attitude from the perspective of all cognitive centers as follows: Iknow provides the available informational support, Iwant the decisional operability, Ilove the info-emotional affectivity with respect to the analyzed/evaluated object/objective, Iam the personal operational capability, Icreate the info-relational/social perspective, Icreated the native/"ancestral" criteria, Ibelieve the trust and confidence.

Therefore, if we have to detect/evaluate and understand, either own or the someone else's attitude, we have to refer specifically to the activity of each cognitive center as an active participant. The detection and evaluation of attitude cannot be therefore limited to the observation of manifested/expressed behavior, although this can be of a helping relevance. The real attitude can be obtained by knowing the contribution of the participating component resulted from each cognitive centers of the mind. Moreover, as it was shown earlier, attitude is not always identical with behavior, as ultimate expression, because if some educational or limitative circumstances by the social norms do not permit explicitly express the intimate attitude, the externally detected behavior could be different of the real attitude.

The evaluation of attitude should be therefore the product of the evaluation of each cognitive center. The evaluation of attitude cannot be limited to the application of a questionnaire only to Iwant, because the response of Iwant is a global reaction, a mediated product of the partial reaction of each participating cognitive center. That is because one or more centers could have a significant contribution in the detriment of the "cool" judicious/theoretical center Iwant itself. The priorities in a decision making of Iwant could be also altered by the intervention of other distinct centers, like for instance Iam, which can claim health-related emergencies or limitations. The nature of the object or objective could require a special kind of judgment, referring specifically to the emotional or only to "mathematical" type of evaluation, so in these cases one specific cognitive center could become more significant in the decision making process. The attitude with respect to an artistic act engages rather the emotional component than the abstract judgment specific for instance to a theoretic problem.

In Figure 1 right side it is shown an example of evaluating diagram taking into account all characteristics discussed above. In this diagram it is represented the participative contribution of each cognitive center to a decision making/evaluation with respect to an object/objective. The questionnaire addressed therefore to a testing participant for the evaluation of Iknow, should refer to the expertise/experience in the related domain. The evaluation is marked on a numerical scale with a maximum of 10 units, as it can be seen in Fig. 1 right side. Three distinct categories of expertise could be qualified at this point: expert (maximum qualification), medium/consumer/user or low/ inexpert level. The emotional component expressing the affective reaction with respect to the object/objective can be quantified in the same manner on a scale of 10 units. The evaluation of the cognitive center Iam can reveal some specific confort/discomfort functions of the organism with respect to the focused objective, classifiable also on a decimal scale. The participation of the center Icreate expresses the social contributory component, highlighting for instance the personal attitude with respect to the gender in the specific context, or particular inter-human relations/positions. Icreated can showcase the personal attitude coming from the particular education and tradition, cultural, social and religious habits, which can be positive or rather negative, as it shown in the diagram from Figure 1.

The diagram represented in the right side of Figure 1 includes the two possibilities of evaluation, positive (favorable) and negative (unfavorable) alternatives. The value zero marks a neutral attitude. As it can be also seen from this diagram, the attitude emanated from the cognitive center Icreate and Icreated could be negative, expressing a pro-active unfavorable attitude against the observed object/objective, whereas the centers Iknow, Ilove, Iam and Ibelieve provide a pro-active positive attitude. The attitude depends on the time, so the evaluation refers actually to a momentary attitude. Marking by Ao(Iwant) the momentary global attitude with respect to an object/objective, then this can be calculated as a function of the contribution of all other cognitive centers, defined specifically by Ao(Iknow), Ao(Ilove), Ao(Iam), Ao(Icreate), Ao(Icreated), Ao(Ibelieve) as following:

Ao $($ Iwant $)=[$ Ao $($ Iknow $)+$ Ao(Ilove $)+$ Ao $($ Iam $)+$ Ao $($ Icreate $)+$ Ao(Icreated) + Ao(believe) $] / 6$

where 6 is the number of the activated centers, used as a mediating factor. 
In relation (1) each contributing attitude must be added including its specific positive or negative sign. Specifically, in the concrete example represented in Figure 1 right side, the numerical values of Ao(Icreate) and Ao(Icreated) will get a negative sign. With the data registered in this diagram, a value of 2.25 situated under the half of the maximum value is obtained (yellow arrow), showing a low level of appreciation of the analyzed objective. If the final value of Ao(Iwant) is positive, the attitude is still favorable even at a low level of appreciation, but if this value is negative, the attitude towards objective is unfavorable. However, more than a global indication, the presented procedure reflecting six distinct components, offers a detailed view on the specific determinant weight of a certain center, so allows a precise detection of the real reason for which a positive or a negative attitude was actually obtained. In the concrete case presented in the diagram of Figure 1, the mentality and social/gender type attitude influence negatively the general attitude. If a personal evaluation on a specific own subject shows a negative or low level result, then a correction to the initial attitude should be applied for a subsequent improvement. This type of analysis precisely detecting the real motif of a negative or low-level result can be applied to any field of activity, allowing a further improvement of the attitude.

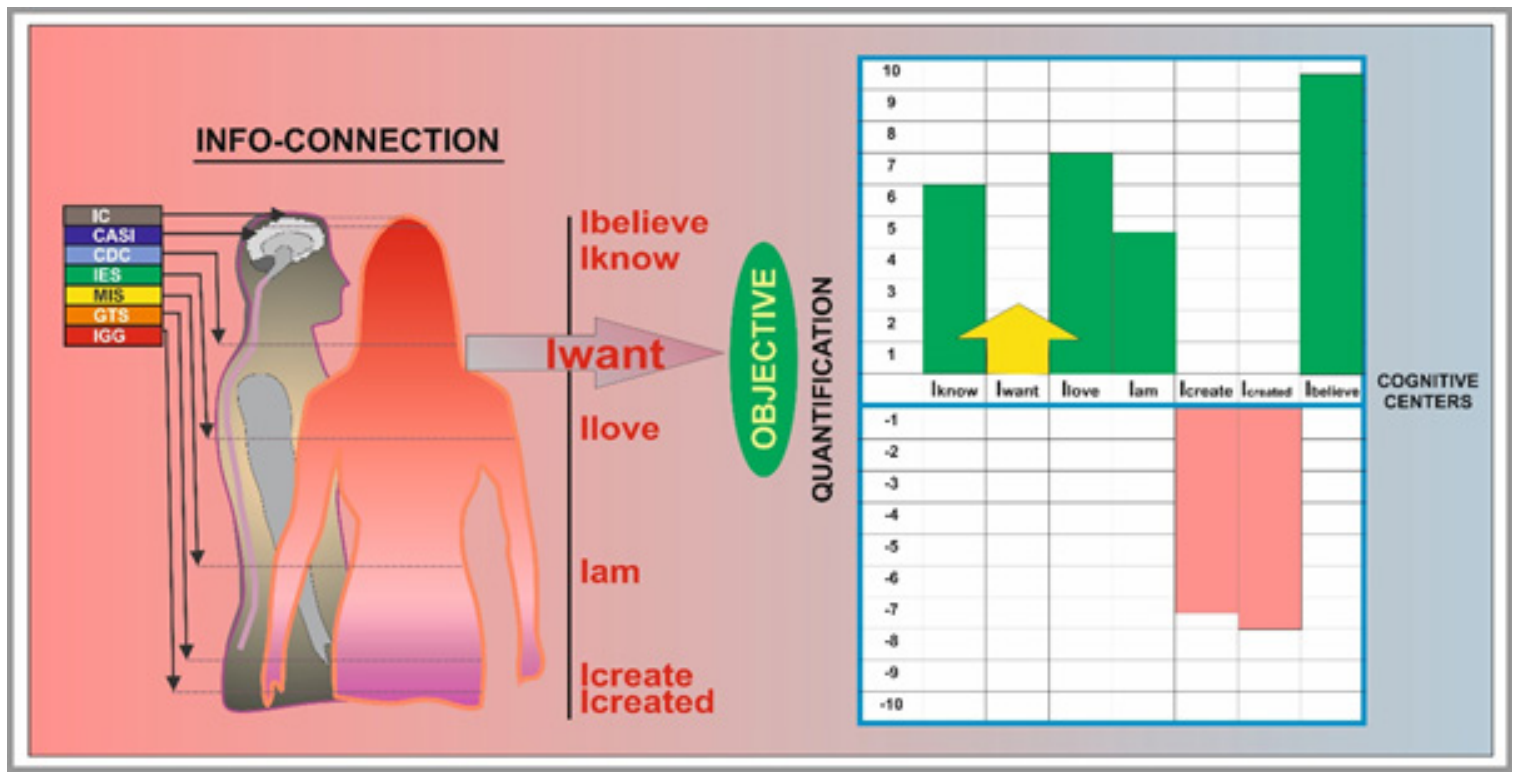

Figure 1: Schematic representation of the operational cognitive centers and their relation with the body functionalities (left side) and the attitude positive/negative evaluation diagram (right side).

In the same manner can be evaluated the momentary behavior Bo with respect to an object/objective, including this time the intimate (nonexplicitly expressed attitude of Iwant) to the general global position, as following:

$\mathrm{Bo}=[\mathrm{Ao}(\mathrm{Iknow})+\mathrm{Ao}($ Iwant $)+\mathrm{Ao}($ Ilove $)+\mathrm{Ao}($ Iam $)+\mathrm{Ao}($ Icreate $)+$ Ao(Icreated) + Ao(believe) $] / 7$

Relations (1) and (2) allow a realistic and full evaluation of own or someone else's attitude and behavior and serves as a professional procedure of evaluation, with applicability in various activity fields, like the quality control and assurance, educational and health domains, rating of the political opinions or satisfaction degree of the customers in services of economic/financial sectors, able to distinguish the detailed reaction of the observers/tested participants from the perspective of all cognitive centers. The evaluation of momentary attitude in various periods of time allows to observe the time evolution of attitude in a particular investigated sector.

The obtained results based on the informational properties of mind and body open a new and valid direction of researches with profitable effects on a better understanding of the human psyche and on society in all adjacent activity areas.

\section{Conclusion}

It was analyzed the attitude from the perspective of the informational relation between mind and body, highlighting on one hand the modular functionality of the human organism, specialized to correlate the material automatic activities with the informational management, and on the other hand the communication with the operative mind working with virtual information and decision making processes, connected to the external and internal reality. There were motivated/ argued the activities of the distinct cognitive centers of the mind, contributing to the final global attitude as a consequence of the modular activity and functions of the human body, centered on metabolic (Iam), genetic transmission (Icreate) and inherited processes (Icreated), which assure the body and species existence, and on the adaptation to the external and internal conditions by accumulation of information (Iknow), selection (Ibelieve), decision (Iwant) and reactive emotivity (Ilove).

It was deduced that the attitude is mainly expressed by Iwant infooutput, representing the activity of the decision making center. The presented procedure is able to evaluate a final global attitude, explicitly revealing the contribution of all other cognitive centers, with positive (favorable) or negative (unfavorable) effect on the attitude, as a function of individual experience (expertise), the affective/emotional reaction, personal comfort/discomfort or health status, gender/social and/or mentality attitude, trust and confidence with respect to the object/objective. There were presented general formulas suitable to calculate both the momentary global attitude and behavior, applicable in a very large range of human activities, from political to social services of industrial, commercial, financial areas, in the field of health and education or in the personal psycho-development processes. The time evolution of attitude could be analyzed applying the procedure evaluation in various time periods.

\section{Acknowledgment}

To my son Adrian Gaiseanu, eminent specialist, son and father, and to his family. To my daughter Ana-Maria Gaiseanu, highly following the best prerogatives in family, life and profession, and to her family. To Traian and Monica and their family.

To corresponding neuropsychology and information science forums and scientists, to the Science and Technology of information in the Romanian Academy. 
Grateful thanks to the Managing Editor and to this Journal for kind invitation to participate with this contributing paper.

In the memory of my loved parents and brother, Professors Emanoil, Florica and Constantin Gaiseanu and of all members of family who were part of my life.

\section{Conflicts of interest}

The author declares there is no conflicts of interest.

\section{References}

1. Perloff Richard M. The Dynamics of Persuasion: Communication and Attitudes in the Twenty-First Century. Lawrence Erlbaum Associates Publishers; London: 2003.

2. Gaiseanu Florin. Language Patterns and Cognitive-Sentient Reality Certainty/Uncertainty in Cognitive-Sentient Exploration of Reality. Stephen Brock Schafer (eds) Chapter in Media Models to Foster Collective Human Coherence in the Psychology. USA; 2019: 49-72.

3. Gaiseanu Florin (2020) What Is Life: An Informational Model of the Living Structures. Biochemistry and Molecular Biology 5(2): 18-28.

4. Gaiseanu Florin (2019) The Informational Model of Consciousness: Mechanisms of Embodiment/Disembodiment of Information. NeuroQuantology 17(4): 1-17.

5. Morris Jerry W, Stuckhardt Michael H (1977) Art Attitude: Conceptualization and Implication. JSTOR 19(1).

6. Wolf Lukas J, Haddock Geoffrey, Maio Gregory R (2020) Attitudes, Oxford Research Encyclopedia of Psychology. Psychology 211-216.

7. Gaiseanu Florin (2020) Multitask Music-Based Therapy Optimization in Aging Neurorehability by Activation of the Informational Cognitive Centers of Consciousness. Gerontol \& Geriatric Stud 6(3): 621-625.
8. Altmann Tanya (2008) Attitude: A Concept Analysis. Nurs Forum 43(3): 144-150.

9. Gaiseanu Florin (2020) Attitude as an Expressible Info-Operational Reaction to a Perceived/Purposed Object/Objective, International Journal on Neuropsychology and Behavioural Sciences 1(1): 12-16.

10. Gaiseanu Florin (2020) Information Based Hierarchical Brain Organization/Evolution from the Perspective of the Informational Model of Consciousness. Archives in Neurology \& Neuroscience 7(5): 1-9.

11. Gaiseanu Florin (2019) Epigenetic Information-Body Interaction and Information-Assisted Evolution from the Perspective of the Informational Model of Consciousness. Archives in Biomedical Engineering \& Biotechnology 2(2): 1-6.

12. Gaiseanu Florin (2019) The Silent Voice of Those Who are no Longer: Transgenerational Transmission of Information from the Perspective of the Informational Model of Consciousness. Gerontology \& Geriatric Studies 5(1): 482-488.

13. Gaiseanu Florin (2020) Informationally-Assisted Equilibrium and Health: Specific ACC Contribution from the Perspective of the Informational Model of Consciousness. EC Psychology and Psychiatry J. 9(5): 37-49.

14. Gaiseanu Florin (2020) Info-Relational Cognitive Operability of the Posterior Cingulate Cortex According to the Informational Model of Consciousness. International Journal of Psychological and Brain Sciences 5(4): 61-68.

15. Gaiseanu Florin (2019) Informational Mode of the Brain Operation and Consciousness as an Informational Related System. Archives in Biomedical Engineering \& Biotechnology 1(5): 1-7. 